\title{
On the critical behavior of the Percus-Yevick equation for nontruncated potentials
}

\author{
J. J. Brey and A. Santos \\ Departamento de Física Teórica, Facultad de Física, Universidad de Sevilla, Apdo. Correos 1065, Sector Sur, Sevilla, Spain
}

(Received 3 August 1984; accepted 17 December 1984)

\begin{abstract}
We present a qualitative analysis on the influence of truncating a long-ranged potential on the critical behavior of a fluid described by the Percus-Yevick equation. It is shown that a nonclassical equation of state for truncated potentials can be compatible with a classical one in the long-range limit. Our main assumption is that the dominant part of the difference between both equations of state is a regular function driven by the asymptotic behavior of the direct correlation function. The results are applied to the case of a Lennard-Jones potential. Comparison with available numerical results is quite satisfactory.
\end{abstract}

\section{INTRODUCTION}

In the last few years, a great attention has been devoted to the study of the behavior predicted in the critical region by the approximate integral equations for fluids. One of the most widely studied is the PercusYevick (PY) equation. Both analytical ${ }^{1-3}$ and numerical $^{4-6}$ studies show that the PY approximation predicts classical values for the critical exponents. However, the exact solution of the PY equation for the so-called stickyhard-sphere model ${ }^{1}$ gives rise to a compressibility equation of state leading to nonclassical scaling functions in the

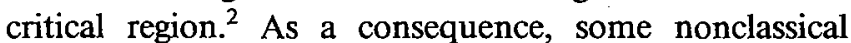
features occur, one of them being a strong asymmetry of the critical isotherm with respect to the critical point. ${ }^{1,2}$ A recent analysis of the PY equation for a lattice gas model with nearest neighbor interaction reveals the same nonclassical features. ${ }^{3}$

For more realistic interactions, numerical procedures are needed to solve the PY equation. From the numerical solution for a truncated Lennard-Jones (TLJ) potential by Henderson and Murphy, ${ }^{4}$ Fishman and Fisher ${ }^{2}$ pointed out the possible existence of a certain asymmetry on the critical isotherm. A stronger asymmetry seems to arise when a potential with an attractive Yukawa tail is considered. ${ }^{5}$ On the other hand, a numerical study for the nontruncated Lennard-Jones (LJ) potential ${ }^{6}$ indicated a purely classical behavior of the PY approximation.

These results support the idea ${ }^{2}$ that the PY approximation exhibits a nonuniversal critical behavior in which, although the critical exponents are always classical, the amplitude ratios, namely the one measuring the "degree" of asymmetry of the critical isotherm, take values dependent on the details (in particular, on the range) of the interaction potential. Our conjecture is that, in the limit of long-ranged potentials, the scaling functions for the equation of state become classical and the critical isotherm is symmetrical. Here, an interaction potential is said to be short ranged if it asymptotically decays faster than any negative power of the distance. So, a potential with an attractive Yukawa tail or any truncated potential has a short range, while the $\mathrm{LJ}$ potential is long ranged.
In this paper we present a simple phenomenological analysis showing the plausibility of the above conjecture. In Sec. II we show that the classical PY equation of state for a long-ranged potential can be compatible with the nonclassical equation of state for a truncated potential, provided that the main difference between both equations of state comes from the asymptotic behavior of the direct correlation function. Moreover, it is possible to relate the critical coordinates and amplitudes for the truncated potential to the ones corresponding to the long-ranged potential. Even more, we are able to derive equations for the change of the critical parameters as the truncation distance increases.

In Sec. III we apply our analysis to the LJ potential. Starting from the values of the critical coordinates and amplitudes corresponding to the TLJ considered by Henderson and Murphy, ${ }^{4}$ we predict the corresponding values for the LJ potential, as well as for other TLJ potentials. Comparison with previous results, when possible, is quite satisfactory, despite the poor accuracy of the values of Ref. 4.

\section{THE ANALYSIS}

The compressibility equation of state reads

$$
\chi^{-1} \equiv \frac{1}{k_{B} T}\left(\frac{\partial P}{\partial \rho}\right)_{T}=1-4 \pi \rho \int_{0}^{\infty} d r r^{2} C(r)
$$

where $P$ is the pressure, $\rho$ is the number density, $T$ is the temperature, $k_{B}$ is the Boltzmann constant, and $C(r)$ is the direct correlation function. The PY approximation consists of closing the Ornstein-Zernike relation by means of the equation

$$
C(r)=g(r)\left[1-e^{\imath(r) / k_{B} T}\right],
$$

where $u(r)$ is the interaction potential and $g(r)$ is the radial distribution function, which tends to unity when $r$ is large. An important property of the PY approximation is that the asymptotic behavior of the direct correlation function $C(r)$ is given by the interaction potential $u(r)$. More concretely, if one assumes that the physical condition 
$g(r) \rightarrow 1$ as $r \rightarrow \infty$ holds in the PY approximation, Eq.

(2) implies

$$
C(r) \simeq-\frac{u(r)}{k_{B} T}
$$

for $r$ large enough, and this will be assumed true even at the critical point.

Now, let $u(r)$ be a long-ranged potential, say the LJ potential. We introduce the potential $u_{R}(r)$ obtained by truncating $u(r)$ at $r=R$, i.e.,

$$
\begin{aligned}
u_{R}(r) & =u(r), & & r \leq R, \\
& =0, & & r>R .
\end{aligned}
$$

The compressibility equation of state for $u_{R}(r)$ is

$$
\chi_{R}^{-1}=1-4 \pi \rho \int_{0}^{R} d r r^{2} C_{R}(r)
$$

$C_{R}(r)$ being the PY direct correlation function for this potential, and where we have taken into account that

$$
C_{R}(r)=0, \quad r>R
$$

At given density and temperature, we have

$$
\chi^{-1}-\chi_{R}^{-1}=-4 \pi \rho \int_{0}^{\infty} d r r^{2} \Delta_{R}(r)
$$

where

$$
\Delta_{R}(r) \equiv C(r)-C_{R}(r) .
$$

The asymptotic behavior of this function is given by

$$
\Delta_{R}(r) \simeq-\frac{u(r)-u_{R}(r)}{k_{B} T} .
$$

To be concrete, let us assume that Eq. (9) holds for $r$ $>r_{0}$ and consider $R>r_{0}$. Then, $\Delta_{R}(r)$ for $r_{0}<r \leq R$ is given by terms that are negligible as compared with $u(r)$ / $k_{B} T$. We write

$$
\begin{aligned}
\Delta_{R}(r) & \simeq 0, & & r_{0}<r \leq R, \\
& \simeq-\frac{u(r)}{k_{B} T}, & & r>R .
\end{aligned}
$$

We expect, for such values of $R, C(r)$, and $C_{R}(r)$ to be very close for $r<R$. More precisely, we assume that

$$
\begin{aligned}
\left|\int_{0}^{R} d r r^{n} \Delta_{R}(r)\right| & \ll\left|\int_{R}^{\infty} d r r^{n} \Delta_{R}(r)\right| \\
& \simeq \int_{R}^{\infty} d r r^{n}\left|\frac{u(r)}{k_{B} T}\right|
\end{aligned}
$$

for $n \geqslant 2$, when $u(r)$ is a long-ranged potential. As a matter of fact, our definition of long-ranged potentials implies that there is a value $n_{0}$ such that the right-hand side of Eq. (11) diverges for $n>n_{0}$. However, although the validity of the inequality (11) for a given $n$ implies that it holds for $n+1$, the reverse is not true.

The assumption (11) for $n=2$ allows us to write

$$
\chi^{-1}-\chi_{R}^{-1} \simeq-\frac{\rho}{k_{B} T} \omega_{R}
$$

where

$$
\omega_{R} \equiv-4 \pi \int_{R}^{\infty} d r r^{2} u(r)
$$

Equation (12) is our main physical ansatz. Its plausibility lies on the long-range character of the interaction potential $u(r)$, and on the behavior (10), which is a consequence of the law (3). For short-range potentials, the right-hand side of Eq. (11) exists for all $n$ and there is no reason to expect inequality (11) to hold. Of course, deeper theoretical and numerical analysis is needed in order to check the validity of Eq. (12).

Notice that we cannot use Eq. (12) to write

$$
\chi_{R^{\prime}}^{-1}-\chi_{R}^{-1} \simeq-\frac{\rho}{k_{B} T}\left(\omega_{R^{\prime}}-\omega_{R}\right),
$$

unless $R^{\prime} \rightarrow \infty$ for a given $R$. The reason is that terms that have been neglected upon writing Eq. (12) can be relevant as compared with the right-hand side of Eq. (14).

Now, suppose that the PY compressibility equation of state for $u_{R}(r)$ takes in the region around the critical point $\left(\rho_{c, R}, T_{c, R}\right)$ the form obtained for sticky hard spheres ${ }^{1,2}$ and also for the lattice gas model with nearest neighbor interaction, ${ }^{3}$ i.e.,

$$
\begin{aligned}
k_{B} T \chi_{R}^{-1} \approx & \left\{\left[B_{R}\left(T-T_{c, R}\right)+A_{R}^{2}\left(\lambda_{R}+1\right)^{2}\left(\rho-\rho_{c, R}\right)^{2}\right]^{1 / 2}\right. \\
& \left.-A_{R}\left(\lambda_{R}-1\right)\left(\rho-\rho_{c, R}\right)\right\}^{2}
\end{aligned}
$$

where $A_{R}, B_{R}$, and $\lambda_{R}$ are critical amplitudes. Along the critical isochore $\rho=\rho_{c, R}$, one has

$$
\begin{aligned}
& k_{B} T \chi_{R}^{-1} \approx B_{R}\left(T-T_{c, R}\right), \\
& \rho=\rho_{c, R}, \quad T-T_{c, R} \rightarrow 0^{+},
\end{aligned}
$$

which corresponds to the classical critical exponent $\gamma$ $=1$. The critical exponent $\delta$ also takes its classical value $(\delta=3)$ since

$$
\begin{aligned}
k_{B} T_{\chi_{R}^{-1}} & \approx 4 A_{R}^{2}\left(\rho-\rho_{c, R}\right)^{2}, \quad T=T_{c, R}, \quad \rho-\rho_{c, R} \rightarrow 0^{+} \\
& \approx 4 \lambda_{R}^{2} A_{R}^{2}\left(\rho-\rho_{c, R}\right)^{2}, \quad T=T_{c, R}, \quad \rho-\rho_{c, R} \rightarrow 0^{-}
\end{aligned}
$$

Nevertheless, the critical isotherm is asymmetrical around the critical point, unless $\lambda_{R}=1$.

The coordinates $\left(\rho_{c, R}, T_{c, R}\right)$ of the critical point depend on the range $R$ of the potential. For TLJ potentials, Watts ${ }^{7}$ showed that both the critical density and temperature increase as $R$ does. Let us define the shifts of the critical coordinates as

$$
\begin{gathered}
x_{R} \equiv \rho_{c}-\rho_{c, R}, \\
t_{R} \equiv T_{c}-T_{c, R},
\end{gathered}
$$

where $\left(\rho_{c}, T_{c}\right)$ is the critical point corresponding to the nontruncated potential. In the spirit of our ansatz (12), we admit that $x_{R}$ and $t_{R}$ are small enough, so that the critical point $\left(\rho_{c}, T_{c}\right)$ and its immediate vicinity lie in the critical region around $\left(\rho_{c, R}, T_{c, R}\right)$, where Eq. (15) holds. In fact, the results reported by Henderson and Murphy for a TLJ potential ${ }^{4}$ show that laws (16) and (17) extend 
until, at least, $T \simeq 1.3 T_{c, R}$ and $\rho \simeq 1.2 \rho_{c, R}$, and the numerical study of Ref. 6 for the LJ potential leads to $T_{c}$ $\simeq 1.01 T_{c, R}$ and $\rho_{c} \simeq 1.04 \rho_{c, R}$.

In summary, Eq. (12) implies that, if Eq. (15) describes the asymptotic PY equation of state in the critical region for a truncated potential, we have

$$
\begin{aligned}
& k_{B} T \chi^{-1} \\
& \approx-\rho \omega_{R}+\left\{\left[B_{R}\left(T-T_{c}+t_{R}\right)+A_{R}^{2}\left(\lambda_{R}+1\right)^{2}\left(\rho-\rho_{c}\right.\right.\right. \\
& \left.\left.\left.+x_{R}\right)^{2}\right]^{1 / 2}-A_{R}\left(\lambda_{R}-1\right)\left(\rho-\rho_{c}+x_{R}\right)\right\}^{2}
\end{aligned}
$$

for the nontruncated potential. The presence of the term $-\rho \omega_{R}$ on the right-hand side of Eq. (20) makes $x_{R}$ and $t_{R}$ to be nonzero, and, therefore, $\chi^{-1}$ becomes a regular function of $\rho$ and $T$ at the critical point $\left(\rho_{c}, T_{c}\right)$. So, near the critical point, Eq. (20) reduces to

$$
k_{B} T \chi^{-1} \approx 4 A^{2}\left(\rho-\rho_{c}\right)^{2}+B\left(T-T_{c}\right),
$$

where

$$
\begin{aligned}
& \left.B \equiv k_{B} T_{c} \frac{\partial \chi^{-1}}{\partial T}\right|_{c}, \\
& \left.4 A^{2} \equiv \frac{1}{2} k_{B} T_{c} \frac{\partial^{2} \chi^{-1}}{\partial \rho^{2}}\right|_{c},
\end{aligned}
$$

and the critical point is given by

$$
\left.\chi^{-1}\right|_{c}=\left.\frac{\partial \chi^{-1}}{\partial \rho}\right|_{c}=0
$$

Equations (24) allow us to obtain $x_{R}$ and $t_{R}$ in terms of the parameters describing the critical region for the truncated potential:

$$
\begin{gathered}
x_{R}=\frac{\omega_{R}}{A_{R}} \frac{1+2 A_{R}\left(\lambda_{R}-1\right)\left(\rho_{c} / \omega_{R}\right)^{1 / 2}}{8 A_{R} \lambda_{R}-\left(\lambda_{R}-1\right)\left(\omega_{R} / \rho_{c}\right)^{1 / 2}}, \\
t_{R}=\frac{\omega_{R}^{2}\left(\lambda_{R}+1\right)^{2}}{B_{R}} \\
\times \frac{16 A_{R}^{2} \lambda_{R}\left(\rho_{c} / \omega_{R}\right)-4 A_{R}\left(\lambda_{R}-1\right)\left(\rho_{c} / \omega_{R}\right)^{1 / 2}-1}{\left[8 A_{R} \lambda_{R}-\left(\lambda_{R}-1\right)\left(\omega_{R} / \rho_{c}\right)^{1 / 2}\right]^{2}} .
\end{gathered}
$$

Strictly speaking, Eq. (25) is an implicit equation for $x_{R}$, as $\rho_{c}=\rho_{c, R}+x_{R}$. In the same way, one gets

$$
\begin{aligned}
B= & \frac{B_{R}}{2 A_{R}} \frac{8 A_{R} \lambda_{R}-\left(\lambda_{R}-1\right)\left(\omega_{R} / \rho_{c}\right)^{1 / 2}}{\left(\lambda_{R}+1\right)^{2}}, \\
4 A^{2}= & \frac{\omega_{R} / \rho_{c}}{4}\left\{1+\frac{B}{B_{R}}\left[16 A_{R}^{2} \lambda_{R}\left(\rho_{c} / \omega_{R}\right)\right.\right. \\
& \left.\left.-4 A_{R}\left(\lambda_{R}-1\right)\left(\rho_{c} / \omega_{R}\right)^{1 / 2}-1\right]\right\} .
\end{aligned}
$$

The asymptotic equation of state (21) is fully classical. In particular, the critical isotherm is symmetrical. In other words, $\lambda_{R} \rightarrow 1$ when $R \rightarrow \infty$, i.e., when $\omega_{R} \rightarrow 0$. In this limit, we also have $x_{R} \rightarrow 0, t_{R} \rightarrow 0, A_{R} \rightarrow A$, and $B_{R} \rightarrow B$.

Equations (25)-(28) allow us to predict the values of $\rho_{c}, T_{c}, A$, and $B$ from the knowledge of $\rho_{c, R}, T_{c, R}, A_{R}$,
$B_{R}$, and $\lambda_{R}$ for a given $R$. This process cannot be reversed, as one of the parameters, say $\lambda_{R}$, would be left undetermined. The physical reason is that we have been able, with the help of assumption (12), to derive Eq. (21) from Eq. (15), but it is impossible to get Eq. (15) from Eq. (21).

Nevertheless, one can study the way in which the critical coordinates and amplitudes behave as $R$ tends to infinity. The structure of Eqs. (25)-(28) suggests writing

$$
\begin{aligned}
& \lambda_{R}=1+\lambda^{(1)} \omega_{R}^{1 / 2}+\lambda^{(2)} \omega_{R}+O\left(\omega_{R}^{3 / 2}\right), \\
& A_{R}=A+A^{(1)} \omega_{R}^{1 / 2}+A^{(2)} \omega_{R}+O\left(\omega_{R}^{3 / 2}\right), \\
& B_{R}=B+B^{(1)} \omega_{R}^{1 / 2}+B^{(2)} \omega_{R}+O\left(\omega_{R}^{3 / 2}\right), \\
& x_{R}=\omega_{R}\left[x^{(0)}+x^{(1)} \omega_{R}^{1 / 2}+O\left(\omega_{R}\right)\right], \\
& t_{R}=\omega_{R}\left[t^{(0)}+t^{(1)} \omega_{R}^{1 / 2}+t^{(2)} \omega_{R}+O\left(\omega_{R}^{3 / 2}\right)\right],
\end{aligned}
$$

where the coefficients are independent of $R$. Substitution of Eqs. (29)-(33) into Eqs. (25)-(28) allows to express all the coefficients in terms of two of them, say $\lambda^{(1)}$ and $\lambda^{(2)}$. The result is

$$
\begin{aligned}
& A^{(1)}=-\frac{A}{2} \lambda^{(1)} \\
& A^{(2)}=\frac{A}{2}\left(\lambda^{(1)^{2}}-\lambda^{(2)}+\frac{3 \lambda^{(1)}}{8 A \sqrt{\rho_{c}}}\right), \\
& B^{(1)}=0 \\
& B^{(2)}=\frac{B}{4}\left(\lambda^{(1)^{2}}+\frac{\lambda^{(1)}}{2 A \sqrt{\rho_{c}}}\right) \\
& x^{(0)}=\frac{\sqrt{\rho_{c}}}{4 A}\left(\lambda^{(1)}+\frac{1}{2 A \sqrt{\rho_{c}}}\right) \\
& x^{(1)}=\frac{\sqrt{\rho_{c}}}{4 A}\left(\lambda^{(2)}-\lambda^{(1)^{2}}\right), \\
& t^{(0)}=\frac{\rho_{c}}{B}, \\
& t^{(1)}=0, \\
& t^{(2)}=-\frac{\sqrt{\rho_{c}}}{8 A B}\left(\lambda^{(1)}+\frac{1}{2 A \sqrt{\rho_{c}}}\right) .
\end{aligned}
$$

In the next section, we will use these expressions to obtain numerical values for the $\mathbf{L J}$ potential.

\section{APPLICATION TO THE LENNARD-JONES $(6,12)$ POTENTIAL}

Let us consider the $\mathrm{LJ}(6,12)$ potential

$$
u(r)=4\left(r^{-12}-r^{-6}\right) \text {, }
$$

where usual units of length and energy have been chosen. The corresponding parameter $\omega_{R}$, defined in Eq. (13), is then

$$
\omega_{R}=\frac{16 \pi}{3} R^{-3}\left(1-\frac{R^{-6}}{3}\right),
$$

so that $\omega_{R}^{1 / 2} \sim R^{-3 / 2}$. 
From a numerical solution of the PY equation for the LJ potential, in which behavior (3) was assumed to hold for $r>r_{0}=5$, a classical critical behavior of the form given by Eq. (21) was found. ${ }^{6}$ The critical coordinates and amplitudes were

$$
\begin{aligned}
& \rho_{c} \simeq 0.288, \\
& T_{c} \simeq 1.320, \\
& A \simeq 2.013, \\
& B \simeq 2.474 .
\end{aligned}
$$

It must be said that these values might be affected by errors because of the numerical algorithm, ${ }^{8}$ namely the choice of $r_{0}$.

On the other hand, Henderson and Murphy ${ }^{4}$ obtained, for a TLJ potential with $R=6$,

$$
\begin{aligned}
& \rho_{c, R} \simeq 0.278, \\
& T_{c, R} \simeq 1.311 .
\end{aligned}
$$

These authors do not quote values of the critical amplitudes. Nevertheless, using their Figs. 3 and 4, we have estimated

$$
\begin{aligned}
& A_{R} \simeq 1.847 \\
& \lambda_{R} \simeq 1.245 \\
& B_{R} \simeq 2.459 .
\end{aligned}
$$

Obviously, not all the figures are significant. As a matter of fact, Fishman and Fisher ${ }^{2}$ estimated $\lambda_{R}=1.28 \pm 0.03$. As our calculations in this section have a mainly qualitative and illustrative character, we are not interested in the study of the propagation of errors coming from the uncertainties of the values (49)-(53).

Although the value $R=6$ is probably not large enough to apply in detail the analysis of Sec. II, we can insert values (49)-(53) into Eqs. (25)-(28) in order to estimate the values predicted for the $\mathrm{LJ}$ potential. The result is

$$
\begin{aligned}
& \rho_{c} \simeq 0.284, \\
& T_{c} \simeq 1.320, \\
& A \simeq 2.027, \\
& B \simeq 2.413 .
\end{aligned}
$$

The agreement with the values (45)-(48) is fairly satisfactory.

Now, we are going to estimate the coefficients in the expansions (29)-(33). By taking advantage from the fact that $B^{(1)}=0$ [Eq. (36)], one could compute $B^{(2)}$ from $B_{R}$ and $B$, provided that terms of order higher than $\omega_{R}$ in Eq. (31) can be neglected. Although for $R=6$ it is $\omega_{R}^{1 / 2}$ $\simeq 0.28$, we have used Henderson and Murphy's results as they are the only ones we are aware of. In this way, one gets

$$
B^{(2)} \simeq 0.5955 .
$$

As $\lambda_{R} \geqslant 1, \lambda^{(1)}$ must be non-negative. The positive root of Eq. (37) is

$$
\lambda^{(1)} \simeq 0.7797 \text {. }
$$

Now, substitution of values (52) and (59) into Eq. (29) yields

$$
\lambda^{(2)} \simeq 0.3589 \text {. }
$$

Finally, Eqs. (34), (35), (38)-(40), and (42) give

$$
\begin{aligned}
& A^{(1)} \simeq-0.7903, \\
& A^{(2)} \simeq 0.5266, \\
& x^{(0)} \simeq 0.08168, \\
& x^{(1)} \simeq 0.003615, \\
& t^{(0)} \simeq 0.1178, \\
& t^{(2)} \simeq-0.01693 .
\end{aligned}
$$

As a test of consistency, let us notice that from Eqs. (61)(66) one reobtains Eqs. (49)-(51).

We can make use of the values (56)-(62) to estimate the critical amplitudes when $R$ is large enough (or, equivalently, $\omega_{R}$ is small enough). Table I shows the results for several values of $R$, as well as the corresponding critical coordinates obtained from Eqs. (25) and (26). Let us emphasize that the values listed in Table $\mathbf{I}$ must be seen as merely indicative, because of the uncertainties of the starting values (49)-(53) and the fact that the values of $R$ here considered are smaller than those for which our analysis is expected to apply. However, comparison with the results obtained from numerical solutions of the PY equation is worthwhile. The agreement in the critical

TABLE I. Critical coordinates and amplitudes for Lennard-Jones potentials with several truncation distances $R$ obtained, by the method described in the text, from the results given in Ref. 4 . The values between brackets correspond to numerical solutions of the Percus-Yevick equation.

\begin{tabular}{cccccc}
\hline \hline$R$ & $\rho_{c, R}$ & $T_{c, R}$ & $A_{R}$ & $\lambda_{R}$ & $B_{R}$ \\
\hline 3.5 & 0.225 & 1.276 & 1.739 & 1.628 & 2.645 \\
& $\left(0.268^{\mathrm{a}}\right)$ & $\left(1.275^{\mathrm{a}}\right)$ & & & \\
5.0 & 0.274 & 1.305 & 1.808 & 1.334 & 2.493 \\
& $\left(0.276^{\mathrm{a}}\right)$ & $\left(1.305^{\mathrm{a}}\right)$ & & & \\
6.0 & $0.278^{\mathrm{b}}$ & $1.311^{\mathrm{b}}$ & $1.847^{\mathrm{c}}$ & $1.245^{\mathrm{c}}$ & $2.459^{\mathrm{d}}$ \\
6.4 & 0.279 & 1.313 & 1.861 & 1.220 & 2.451 \\
& $\left(0.282^{\mathrm{e}}\right)$ & $\left(1.316^{\mathrm{e}}\right)$ & & & $\left(2.540^{\mathrm{f}}\right)$ \\
8.0 & 0.282 & 1.316 & 1.902 & 1.153 & 2.432 \\
10.0 & 0.283 & 1.318 & 1.934 & 1.107 & 2.423 \\
15.0 & 0.284 & 1.319 & 1.974 & 1.057 & 2.416 \\
$\infty$ & 0.284 & 1.320 & 2.027 & 1.000 & 2.413 \\
& $\left(0.288^{\mathrm{g}}\right)$ & $\left(1.320^{\mathrm{g}}\right)$ & $\left(2.013^{\mathrm{g}}\right)$ & $\left(1.000^{\mathrm{B}}\right)$ & $\left(2.474^{\mathrm{g}}\right)$ \\
\hline \hline
\end{tabular}

${ }^{a}$ Given in Ref. 7.

${ }^{b}$ Given in Ref. 4.

c Estimated from Fig. 4 of Ref. 4.

d Estimated from Fig. 3 of Ref. 4.

${ }^{\circ}$ Given in Ref. 9.

' Estimated from Fig. 2 of Ref. 9.

${ }^{8}$ Given in Ref. 6. 
temperature is fairly good, but there is a systematic deviation in the critical density. Comparison in the critical amplitudes is hardly possible, as they have been rarely studied. Apart from the values for the nontruncated potential, commented before, it is quite good the agreement in $B_{R}$ for $R=6.4 .^{9}$

In summary, we have shown that in the PY approximation a nonclassical equation of state in the critical region for a truncated potential may be consistent with a fully classical one in the long range limit. The main point in our analysis is that the asymptotic behavior (3) is inherent to the PY approximation at any thermodynamical state. Thus, if the interaction is long ranged, it is justified to expect the right-hand side of Eq. (7) to be a regular function of density and temperature, even at the critical point. This suffices to prove the above consistency. In order to carry out explicit calculations we have assumed the validity of Eq. (12).

Therefore, the PY approximation seems to present a nonuniversal critical behavior, in such a way that the critical isotherm amplitude ratio depends on the interaction range, tending towards unity when the range becomes infinity. We have explicitly studied this for truncated Lennard-Jones potentials and found a satisfactory agreement with previous results. Nevertheless, accurate numerical solutions for several truncation distances are required in order to check the reliability of the analysis presented here.

${ }^{1}$ R. J. Baxter, J. Chem. Phys. 49, 2770 (1968).

${ }^{2}$ S. Fishman and M. E. Fisher, Physica A 108, 1 (1981).

${ }^{3}$ A. Parola and L. Reatto, Physica A 125, 255 (1984).

${ }^{4}$ D. Henderson and R. D. Murphy, Phys. Rev. A 6, 1224 (1972).

${ }^{5}$ F. Gallerani, G. Lo Vecchio, and L. Reatto, Phys. Rev. A (to be published).

6 J. J. Brey, A. Santos, and F. Romero, J. Chem. Phys. 77, 5058 (1982).

${ }^{7}$ R. O. Watts, J. Chem. Phys. 48, 50 (1968).

${ }^{8}$ J. J. Brey and A. Santos, J. Chem. Phys. 79, 4652 (1983).

${ }^{9}$ M. I. Guerrero, G. Saville, and J. S. Rowlinson, Mol. Phys. 29, 1941 (1975). We must point out that, upon estimating $B_{R} \simeq 2.540$, we have taken into account a printing error in the legend to Fig. 2. The vertical axis should read $\left(\rho k_{B} T\right)^{-1}(\partial P / \partial \rho)_{T}$. We thank J. S. Rowlinson for correspondence about this point. 